\title{
LA GAMIFICACIÓN COMO RECURSO DIDÁCTICO PARA LA ENSEÑANZA DE LA HISTORIA
}

\section{GAMIFICATION AS A TEACHING RESOURCE FOR TEACHING HISTORY}

\author{
María Elena Cascante Gómez* \\ Roberto Granados Porras**
}

Fecha de recepción: $31 / 07 / 2018$

Fecha de aceptación: 21/08/2018

Resumen: El presente trabajo describe la importancia de la gamificación como un elemento clave en el proceso de enseñanza-aprendizaje, con el fin de generar un nuevo conocimiento histórico en el marco de la mediación pedagógica. Este enfoque pretende atender una necesidad específica que se debe fortalecer por medio de la renovación e innovación en la didáctica que se emplea en las clases de Historia por parte de los docentes en formación. Se define la gamificación, como un proceso de alfabetización en la competencia digital para la apropiación de recursos tecnológicos y su incorporación en los contextos de aprendizaje mediante procesos pedagógicos. La gamificación incorpora elementos del diseño del juego para aprovecharlos en el proceso de enseñanza-aprendizaje, pero estos no se utilizan por sí mismos, sino que se toman algunos de sus principios o mecánicas como por ejemplo los puntos, los incentivos, la narrativa, la retroalimentación, el reconocimiento o la libertad a equivocarse como medios para generar un aprendizaje significativo. El estudio describe una serie de valoraciones obtenidas de un grupo de estudiantes que está en la mitad de la carrera del Bachillerato de la Enseñanza de los Estudios Sociales y la Educación Cívica (BEESEC) de la Universidad Nacional de Costa Rica sobre sus conocimientos e impacto en la mediación pedagógica que podría generar la gamificación como estrategia de enseñanza-aprendizaje.

Palabras claves: gamificación; juego pedagógico; habilidad pedagógica; mediación pedagógica; enseñanza de la historia.

Abstract: The present work describes the importance of gamification as a key element in the teaching-learning process in order to generate a new historical knowledge within the framework of pedagogical mediation. This approach aims to meet a specific need that must be strengthened through renewal and innovation in the teaching that is used in History classes by

\footnotetext{
* Costarricense. Máster en Pedagogía de la Diversidad y Licenciada en la Enseñanza de los Estudios Sociales por la Universidad Nacional, Costa Rica (UNA). Profesora del Colegio Marista de Alajuela, Costa Rica. Académica e investigadora de la División de Educología de la UNA desde 1995. Correo electrónico: lodelena@yahoo.com

** Costarricense. Máster en Historia Aplicada por la Universidad Nacional, Costa Rica (UNA) y Licenciado en Estudios Sociales y Educación Cívica por la Universidad Estatal a Distancia, Costa Rica (UNED). Profesor e investigador de la Escuela de Historia de la UNA. Coordinador del Proyecto Gestión de la Calidad BEESEC. Correo electrónico: c.roberth@gmail.com
} 
teachers in training. The Gamification is defined as a literacy process in digital competence for the appropriation of technological resources and their incorporation in learning contexts through pedagogical processes. The gamification incorporates elements of game design to take advantage of them in the teaching-learning process, but these are not used by themselves, but rather some of their principles or mechanics are taken as points, incentives, narrative, feedback, recognition or the freedom to make mistakes as a means to generate meaningful learning. The study describes a series of evaluations obtained from a group of students who is in the middle of the career of the Social Studies and Civic Education Teaching of the National University of Costa Rica on their knowledge and impact on the pedagogical mediation that gamification could generate as a teaching-learning strategy.

Keywords: Gamification; Pedagogical Game; Teaching Skills; Pedagogical Mediation; History Education.

\section{Presentación}

El presente trabajo plantea el reto de una estrategia de enseñanzaaprendizaje basada en la gamificación, con el fin de generar un nuevo conocimiento histórico en el marco de la mediación pedagógica, partiendo de una necesidad específica, la renovación e innovación en la didáctica que se emplea en las clases de Historia a los docentes en formación. Se propone la gamificación, entendida como la forma para potenciar procesos de aprendizaje basados en el uso de una serie de recursos tecnológicos como lo son el juego, los Blogs, entre otros, que potencian el desarrollo de procesos de enseñanza y aprendizaje significativos, y a la vez, favorecen la motivación por los contenidos históricos.

Con el paso de los años, enseñar Historia se ha vuelto una tarea difícil. En algunos niveles de enseñanza, el estudiantado presenta un rechazo hacia sus contenidos, pues se considera como una asignatura memorística y aburrida. Pero esta percepción o realidad no es culpa del estudiantado, sino de quien imparte las clases que lo sigue haciendo con el mismo método empleado décadas atrás. Al respecto, Taracena (2015) menciona: 
En la actualidad no resulta extraño escuchar que la historia es aburrida. Este es un reclamo generalizado que ha alcanzado atributo de sentido común al señalar la enseñanza de la historia como una materia dedicada a memorizar datos (p. 11).

Por lo tanto, esta etiqueta de la enseñanza de la Historia puede afectar metodológicamente la función social de su enseñanza, pues existe una negación y apatía en todos los niveles donde se imparte. Siguiendo con la propuesta de Taracena (2015), el autor menciona que la afirmación de "historia aburrida" se ha convertido en un estigma y lo más problemático de esta situación, es la desaprobación y negación de la utilidad de la historia para enfrentar y proponer soluciones a los problemas de la vida práctica. Además, en otros contextos de aprendizaje como a nivel universitario, la Historia se relaciona con un conocimiento erudito, siendo una afirmación poco acertada que desde los planteamientos de la presente investigación se rechaza.

Ahora bien, el problema de la enseñanza de la Historia también puede radicar en la didáctica, pues se coloca al "maestro/a transmitiendo conocimientos unilateralmente, manteniendo en situación de pasividad al alumno" (Taracena, 2015, p. 11). Por eso, en la actualidad se habla de una renovación de la didáctica en las Ciencias Sociales, pues existe la misma situación que en la Historia, ya que se sitúa al estudiantado como "[...] un receptor pasivo de contenidos y en los que el libro de texto es el dispositivo pedagógico que guía la mayoría de las decisiones del docente” (Feliu, Vallés y Cardona, 2016, p. 2).

\section{Componentes teóricos}

Como primer elemento teórico se presenta la gamificación que incorpora elementos del diseño del juego para aprovecharlos en el proceso de enseñanzaaprendizaje, pero este no se utiliza por sí mismo, sino que se toman algunos de 
sus principios o mecánicas como los puntos, los incentivos, la narrativa, la retroalimentación, el reconocimiento o la libertad a equivocarse como medios para generar un aprendizaje significativo.

La relación entre educación y juego es estrecha, y su estudio se desarrolla desde hace algunas décadas. Fandos y González (2013) mencionan que autores como Huizinga (1987) y Caillois (2001) "son pioneros en la redefinición del concepto juego y su relación con los nuevos desarrollos tecnológicos" y señalan que uno de los "elementos fundamentales del juego es la relación existente entre las reglas establecidas y la experiencia del jugador con amplio margen de maniobrabilidad dentro de esas normas; o lo que es lo mismo, el equilibrio entre reglas y libertad" (p. 2).

Fandos y González (2013) argumentan que la gamificación se empieza a utilizar en el 2008 con cierta frecuencia entendida como el uso de elementos de juego en entornos no de juego. A la vez, Mora (2013) citado por Llopis y Balaguer (2016), define la gamificación como una metodología en la cual el profesorado diseña una actividad de aprendizaje -analógica o digitalintroduciendo elementos del juego -insignias, límite de tiempo- para activar el pensamiento -retos, competición- con el fin de enriquecer la experiencia y dirigir o modificar el comportamiento.

En los procesos de enseñanza y aprendizaje, el juego es una herramienta pedagógica que se puede adaptar a cualquier nivel educativo. Llopis y Balaguer (2016) anotan que cuando gamificamos en educación llevamos al aula los elementos y características principales de juegos para implicar al alumnado y ofrecerle una forma de aprendizaje más cercana a su lenguaje y a su forma de entender el mundo. Los autores apuntan que la gamificación no consiste en disfrazar unos contenidos aplicando una serie de puntos y recompensas, sino que considera al usuario el centro y motivo del proceso, y por lo tanto, el 
alumnado debe sentirse plenamente involucrado y divertirse a la vez que consigue objetivos educativos dentro del proceso de mediación.

Para la experta en diseño de videojuegos Jane McGonigal (2011) citada por EduTrends (2016), en el mundo de los juegos, aun sin ser lo suficientemente bueno, el jugador puede tener una experiencia muy divertida. Pues los juegos ofrecen un espacio seguro para fallar y aprender, retar a los usuarios y proveerles de retroalimentación inmediata y de conexiones sociales. Los diseñadores de juegos se han especializado en cómo hacer la experiencia del ambiente de juego tan memorable y adictiva que, incluso cuando los usuarios han dejado de jugar, siguen pensando en estrategias de solución para los retos que se les presentan (Kiang, 2014, citado por EduTrends, 2016).

En cuanto a la enseñanza de la Historia se debe proponer la formación del pensamiento histórico que se convierta en nuevas destrezas y habilidades en el estudiantado. De acuerdo con González, Pagès y Santisteban (2009), las fuentes históricas primarias y secundarias tienen un gran valor educativo para el desarrollo del pensamiento histórico como una competencia necesaria en el estudiantado. Entonces, el nuevo profesorado en Historia debe proponer el empleo de este tipo de fuentes, dado que esto acabaría con el abuso de manuales que generan poco debate y destrezas cognitivas en el estudiantado, y limitan el conocimiento de hechos históricos valiosos que deben conocer los estudiantes para generar un aprendizaje integral de la historia y no desde la visión de un libro que parcializa la información.

Asimismo, la enseñanza de la Historia propone el rescate de la memoria, tanto de procesos históricos nacionales, como de los mundiales considerados importantes para conocer la evolución de la civilización de la humanidad. Dentro de la memoria histórica sobreviven procesos y experiencias que han hecho a la humanidad acumular conocimiento y avanzar. Conforme avanzó la evolución cultural, se hizo más difícil recuperar la memoria, por lo tanto, el 
registro del pasado debió pasar por una transformación. Por ejemplo, en el ámbito mundial se encuentran pinturas rupestres, petroglifos, templos con murales, códices, quipus o bóvedas con restos humanos que posibilitaron recuperar e interpretar hechos históricos considerados como importantes para comprender la evolución del ser humano.

Por consiguiente, la presente investigación toma el empleo de la tecnología en la enseñanza de la Historia como un aliciente para mejorar la metodología con que se tratan los temas históricos en los contextos de enseñanza-aprendizaje. Respondiendo a esta situación, la gamificación educativa busca evidenciar la desmotivación, la falta de compromiso de los estudiantes con los abordajes teórico-metodológicos de los temas históricos que se desarrollan en el aula. Rose y Meyer (2002), citados por Llopis y Balaguer (2016), plantean que los docentes se han de preguntar qué formas diferentes de presentar el contenido existen; cómo pensar en diferentes estrategias que hagan referencia a formas de jugar y aprender, y finalmente, por qué, es decir, los alicientes por los cuales el alumnado se interesa y comprende la tarea.

En la actualidad, muchos de los aprendizajes son mediados por la tecnología, es por eso que la enseñanza de la Historia presenta amplias ventajas al contar con diversas herramientas que colaboran en la mediación docente. Basado en lo anterior, Collazo y de Ranalletti (2015) citando a Meyer (2001), señalan que los aprendizajes multimedia se caracterizan por "seleccionar o diseñar herramientas pedagógicas con empleo de recursos multimedia y digitales" (p. 10).

La renovación metodológica en la enseñanza de la Historia propone que se vivencien los aprendizajes y que el estudiantado se identifique con los procesos que se estudian, por lo tanto, su enseñanza se debe adaptar a la actualidad y en cierta medida estar relacionada con el empleo de herramientas tecnológicas para generar nuevas habilidades. Además, parte importante de 
este proceso es la vinculación de la comunidad estudiantil en el diseño de las lecciones, es necesario que se sientan partícipes del proceso y que como nuevo profesorado en Estudios Sociales puedan opinar sobre la mejor forma de construir el conocimiento.

Por consiguiente, la enseñanza de la Historia se debe adaptar al avance tecnológico que se desarrolla en la actualidad, dado que repercute directamente en los sistemas educativos y proporcionan nuevos canales de información y comunicación, sobre todo vinculados a dispositivos informáticos. Aunque otros relacionan este desarrollo tecnológico con un proceso de mercado y globalización, desde el ámbito educativo se busca la aplicación de aula y que el estudiantado pueda desarrollar habilidades que lo lleven a responder problemas prácticos de la vida cotidiana y participar en una sociedad con altas competencias tecnológicas.

Por lo tanto, la tecnología educativa de acuerdo con Fallas y Trejos (2013): "Se refiere al estudio y práctica ética de facilitar el aprendizaje y mejorar el desempeño, creando, usando y administrando procesos y recursos tecnológicos apropiados" (p. 53). En consecuencia de lo anterior, el cuerpo docente en la enseñanza de la Historia debe plantearse el reto de emplear los recursos tecnológicos que mejor se adapten a su temática y así proponer clases que modelen como ejemplos de innovación educativa.

Ahora bien, dentro de esta nueva propuesta con enfoque tecnológico no se debe dejar de lado la mediación pedagógica que se considera como el acompañamiento que lleva a cabo el profesorado con el estudiantado, caracterizado por ser un guía que estimule los aprendizajes significativos con actividades de clase que se ajusten a la realidad del estudiantado. Es decir, el mediador es la persona que, al relacionarse con otra u otras, según Ferreiro y Vizoso (2008) propicia el paso del sujeto que aprende de "un estado inicial de no saber, poder o ser, a otro cualitativamente superior de saber, saber hacer y, lo 
que es más importante, ser" (p. 76). El mediador, por lo tanto, debe propiciar el aprendizaje, iproponer el desarrollo de potencialidades y corregir funciones cognitivas deficientes; mueve, en términos vigotskianos, al sujeto que aprende en su zona de desarrollo potencial (Ferreiro y Vizoso, 2008, p. 76).

En la enseñanza de la Historia existen problemas de mediación. El profesorado, en muchos casos, se ha convertido en un transmisor vertical de contenidos y como consecuencia las actividades de clase no se ajustan a las necesidades educativas del colectivo discente. Por eso, desde la mediación pedagógica en Historia, se necesita una renovación y un compromiso del profesorado, para llegar a una práctica pedagógica adecuada y que estimule el aprendizaje de estos contenidos con actividades que se planteen como verdaderos retos.

En la actualidad, en la mediación histórica se incorporan diferentes tecnologías, pero en algunos casos erróneamente. Por eso, se pretende que el empleo de la tecnología signifique un cambio cualitativo que impulse una vivencia más cercana en el desarrollo de los contenidos de Historia y que estos se apliquen socialmente. Como lo señala Gros, (2008) citado por Llopis y Balaguer (2016), las TIC permiten ampliar espacios de aprendizaje más allá del aula, al tiempo que acercan los contenidos al alumnado, al darles un formato atractivo, familiar, intuitivo y automatizado.

Por último, se conceptualiza sobre los aprendizajes significativos, que se relacionan directamente con una adecuada mediación de los contenidos de Historia. El estudiantado, en el transcurso del desarrollo académico, recibe una cantidad considerable de contenidos en este ámbito, pero no necesariamente todos generan un aprendizaje adecuado y funcional. Es acá donde se cita de nuevo el papel del profesorado que se encarga de generar una mediación pedagógica adecuada y que sea funcional en todas las etapas y procesos del aprendizaje. 
En este punto se citan los elementos teóricos del constructivismo que argumenta "[...] los individuos viven en el mundo de sus propias experiencias personales y subjetivas. Siendo el individuo quien impone significado sobre el mundo, en vez del mundo imponerle su significado, construyendo una visión personal de la realidad" (Karagiorgi y Symeou, 2005, citados por Cenich y Santos, 2010, p. 8). Por lo anterior, para generar aprendizajes significativos en Historia, el profesorado no debe fungir como un transmisor de contenidos sino como un mediador que promueva un intercambio y debate constante que propicie el aprendizaje como un fenómeno de construcción personal antes de un proceso de transmisión, destacando el rol activo del estudiantado.

\section{Abordaje metodológico}

El trabajo se basó en el análisis de diferentes argumentaciones sobre los principios teóricos y metodológicos de la gamificación como una propuesta renovadora de la enseñanza de la historia y su impacto en los estudiantes del BEESEC de la UNA. Considera a la vez los aportes y opiniones de los estudiantes de segundo y cuarto niveles de la carrera del BEESEC en el 2017, en relación a las concepciones sobre la gamificación como estrategia de aprendizaje y cómo esta puede ser una experiencia de aula que ayude a cambiar las concepciones sobre la metodología empleada en la enseñanza de la historia, para lo cual se utilizaron dos entrevistas semiestructuradas en línea, donde los participantes anotaron sus valoraciones e interpretaciones sobre la gamificación como enfoque innovador de aprendizaje. 


\section{Resultados}

En este apartado, se presentan las valoraciones que generaron los estudiantes que cursan la carrera del BEESEC de la UNA con ayuda de dos entrevistas semiestructuradas en línea, con respecto al concepto de gamificación. Valoraciones generales que incluyen los siguientes aspectos: cómo se genera aprendizaje con ayuda de la gamificación como recurso de aprendizaje, las diferencias entre el juego y la gamificación, la mediación pedagógica alternativa e innovación en el aula que involucra nuevos roles de los participantes del proceso de aprendizaje de la historia.

Estas valoraciones responden a interpretaciones generales de los estudiantes, debido a que el conocimiento o aplicación de la gamificación en los procesos de mediación están limitados a la falta de sistematización o puesta en práctica de actividades docentes donde entra en juego la gamificación, ya que como se menciona en líneas arriba, la población que participó en responder la entrevista son estudiantes que están en mitad de su formación.

\section{Conceptualización de la gamificación por parte del estudiantado}

Como se analizó, la gamificación no implica incorporar elementos del juego sin propiciar aprendizajes que sean significativos. El estudiantado tiene la oportunidad de experimentar con elementos que involucren el apoyo tecnológico, pero también, con propuestas de aprendizaje provenientes de una metodología tradicional con un nuevo enfoque didáctico. Ahora bien, cuando se solicitó al estudiantado si podrían definir qué se entiende por gamificación, las siguientes son algunas de las respuestas: "Es un concepto para introducir principios del juego a la educación”; “[...] es la utilización de juegos en el área 
pedagógica. Para extraer el mayor provecho en el proceso de enseñanza y aprendizaje"; "aplicar conocimientos sobre juegos de diferente índole, para enseñar de forma más moderna los distintos contenidos de estudio" (Entrevista No. 2, ítem 1, 13 de noviembre, 2017).

Sin duda, la gamificación es un concepto relativamente nuevo en la enseñanza de la Historia. Es así como el profesorado se acerca de forma pausada a esta propuesta de enseñanza-aprendizaje, mientras que otros tratan de clarificar el aporte de esta estrategia didáctica. Con las definiciones anteriores, queda claro que el estudiantado conoce de forma somera el significado de este concepto, la mayoría opina que es la incorporación del juego en el área educativa, pero se presenta de una manera segmentada, pues desde este enfoque de aprendizaje se puede proponer una clase, una unidad o un curso completamente gamificado.

Por lo tanto, se visualiza la importancia de los diferentes espacios de aprovechamiento que la gamificación puede otorgar a la enseñanza de la Historia. Tomando en cuenta que, los contextos de aprendizaje de la Historia aún se encuentran caracterizados por el tradicionalismo y sobre todo por el aburrimiento del estudiantado, que son alimentados por un estereotipo social que la historia debe ser memorística y que de poco sirve a nivel social. Pero es necesario mencionar que, algunos de estos estereotipos son alimentados por el mismo profesorado que no busca una renovación didáctica en su metodología de enseñanza.

Esta situación la expresa el estudiantado del cuarto nivel del BEESEC, pues al entrar en contacto con los ambientes escolares por medio de su práctica docente profesional en el sistema educativo nacional por un período de al menos cuatro a cinco meses, percibe que el conformismo es una de las problemáticas que más afecta al profesorado en ejercicio. Pero también existen otras como la negativa a la actualización docente, la sobrecarga de trabajo y los 
problemas relacionados con la mediación pedagógica. Esta situación se refleja en el siguiente gráfico:

\section{Gráfico 1}

Problemáticas más frecuentes en el profesorado activo de EESEC según la percepción del estudiantado de cuarto nivel del BEESEC (2017)

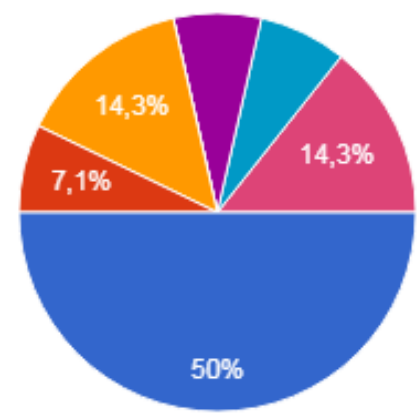

Conformismo

Mediación pedagógica

Sobre carga de trabajo

Grupos numerosos

Actividades extracurriculares

Pocos espacios de actualización docente

Negativa a la actualización docente Limitada comprensión del currículo vigente

Fuente: Elaboración propia. Entrevista No. 1, ítem 3, 4 de octubre, 2017.

Ahora bien, cuando se le preguntó al estudiantado que argumentara sus respuestas se expresó lo siguiente:

1. Los docentes ya no buscan como salir de sus clases magistrales. En vez de innovar y ser más docentes, las evaluaciones son las mismas de hace 30 años, 3 preguntas de desarrollo, que requieren 1 hora por cada respuesta, es una manera muy desgastante y más liberadora para el profesorado.

2. La negativa de la actualización docente es una de las problemáticas que afecta directamente los procesos de enseñanza y aprendizaje. Paralelamente se encuentra el conformismo, pues este impide la actualización misma. Con los diferentes retos de la educación actual, si no se actualiza el docente, se crearía una problemática mayor.

3. Porque los profesores deciden caer en el sistema educativo que propone el Estado, el cual es solo a su conveniencia, dejando de lado 
la criticidad en los estudiantes. Esto ocurre porque es más fácil poder cumplir con un programa ya hecho, en lugar de buscar desarrollar capacidades en los alumnos (Entrevista No. 1, ítem 4, 4 de octubre, 2017).

Por consiguiente, dichas problemáticas son similares a épocas anteriores y la actualización docente no necesariamente está presente en la experiencia profesional. El conformismo asociado a la estabilidad laboral es alto y los enfoques innovadores de aprendizaje como el que se analiza en este trabajo, no forman parte de las actividades de clase en la enseñanza de la Historia.

\section{La gamificación como enfoque para generar aprendizajes significativos en la enseñanza de la Historia}

Las valoraciones de los estudiantes sobre el proceso de enseñanzaaprendizaje, y en especial la generación de aprendizajes en la enseñanza de la historia con ayuda de la gamificación como apoyo docente, inicialmente señalan que la gamificación es un elemento que propicia la participación activa y consciente en el aprendizaje de los datos y hechos históricos, potenciando la posibilidad de adoptar diferentes posiciones y argumentaciones, dejando de lado el uso exclusivo de la memoria como única forma de aprender hechos y acontecimientos históricos. Lo anterior se evidencia en sus respuestas, al anotar que la gamificación es "sumamente útil porque es una manera de que los estudiantes apliquen lo que se les está enseñando y no solo lo memoricen, además tiene una parte importante en la motivación ya que es una técnica innovadora que involucra de lleno al estudiante" (Entrevista No. 2, ítem 2, 13 de noviembre, 2017).

Además, la motivación que se genera con las experiencias de gamificar los procesos de aprendizaje y es un factor importante para salir de entornos de aprendizaje tradicionales y comunes, al estudiante se le hace difícil relacionar 
los hechos históricos con elementos cotidianos. Llopis y Balaguer (2016) señalan que cuando gamificamos en educación, llevamos al aula los elementos y características principales de los juegos para implicar al alumnado y ofrecerle una forma de aprendizaje más cercana a su lenguaje y a su manera de entender el mundo, y en la que su satisfacción es particularmente relevante (p. 86).

Lo anterior, relacionado con la motivación, lo respaldan los estudiantes en sus valoraciones al anotar que "se sale de lo común y lo tradicional. Se le puede facilitar más a un estudiante relacionar aspectos relacionados con la materia a su cotidianidad mediante la utilización de juegos" (Entrevista No. 2, ítem 2, 13 de noviembre, 2017). Las estrategias de aprendizaje novedosas requieren de nuevos enfoques sobre la necesidad de generar habilidades propias de la enseñanza de la historia, como se señaló en páginas anteriores, se debe potenciar en el estudiantado roles activos y una mediación que les incite a plantearse retos y soluciones a los problemas presentes. Es decir, sale de la metodología tradicional de la enseñanza de la Historia.

$\mathrm{Al}$ respecto, los estudiantes señalan que el uso de los recursos tecnológicos que se promueve con la gamificación facilita, "el razonamiento, la lógica y la búsqueda de resolución de problemas por medio de la investigación propia, y estos son muy útiles y aplicables en el aula" (Entrevista No. 2, ítem 3, 13 de noviembre, 2017). En la siguiente tabla, se recopilan las apreciaciones del colectivo estudiantil sobre la gamificación y los aprendizajes significativos: 


\section{Tabla 1}

\section{Utilidad de la gamificación para generar aprendizajes significativos en la enseñanza de la Historia según las apreciaciones del estudiantado del segundo nivel del BEESEC (2017)}

\begin{tabular}{|c|l|}
\hline \multirow{2}{*}{ Nivel } & \multicolumn{1}{|c|}{ Valoraciones en torno a la gamificación } \\
\hline \multirow{5}{*}{$\begin{array}{l}\text { Segundo } \\
\text { nivel del } \\
\text { BEESEC }\end{array}$} & $\begin{array}{l}\text { Es una buena herramienta didáctica. } \\
\text { como el estudiante pueden caer en una zona de comodidad, } \\
\text { desarrollando prácticas monótonas y poco significativas. }\end{array}$ \\
\cline { 2 - 3 } & $\begin{array}{l}\text { estudiantes apliquen lo que se les está enseñando y no solo lo } \\
\text { que es una técnica innovadora que involucra de lleno al estudiante. }\end{array}$ \\
\cline { 2 - 3 } & $\begin{array}{l}\text { La motivación es la principal herramienta que tanto el docente como } \\
\text { el estudiante deben poseer de forma asertiva para realizar un } \\
\text { aprendizaje significativo. }\end{array}$ \\
\hline
\end{tabular}

Fuente: Elaboración propia. Entrevista No. 2, ítem 3, 13 de noviembre, 2017.

\section{Medición pedagógica alternativa y cambio de roles}

La creación de espacios de aprendizaje significativos y duraderos, con el desarrollo de la gamificación como recurso de aprendizaje, es un aspecto cada vez más común, ya que en la medida que se incorporan las tecnologías de la información al ámbito educativo, se hace más habitual generar entornos de enriquecimientos de los procesos de aprendizaje, así entran en la escena de la mediación y en particular de la historia, nuevos y mejorados métodos y técnicas de mediación, que hacen que los profesores y los estudiantes jueguen nuevos roles. Al respecto, los estudiantes señalan que "la búsqueda de alternativas pedagógicas que estén más acordes con el contexto educativo y que aprovechen los TICs para la producción de conocimiento (Entrevista No. 1, ítem 7, 4 de 
octubre, 2017). Lo anterior es clave para entender que el llamado es a generar entornos significativos de aprendizaje, que potencien las habilidades con el fin de ayudar a entender los procesos históricos, con nuevos enfoques y la puesta en práctica, a través de la utilización de recursos tecnológicos que sean motivantes para acercarnos a los intereses y realidades propias del estudiantado.

Las alternativas significativas de aprendizaje plantean la necesidad de generar cambios de roles en los participantes dentro de la dinámica de la enseñanza y en particular de la enseñanza de la historia, es importante eliminar la concepción tradicional del docente como informador de los datos históricos y el estudiante como un ente pasivo que recibe la información. Es decir, utilizar la gamificación en el proceso de aprendizaje, introduciendo formas novedosas y demandantes en la realidad educativa, claro está, sin perder el docente su papel de facilitador en la mediación. Al respecto, los estudiantes señalan que el papel del docente será "de mediador y de guía, no de

dador del conocimiento como mayormente se cree" (Entrevista No. 2, ítem 11, 13 de noviembre, 2017).

\section{Diferencias entre el juego y la gamificación}

Como se mencionó anteriormente, la gamificación incorpora elementos del juego, pero este debe buscar una aplicación didáctica. Según Llopis y Balaguer (2016), "El juego en el aula es una herramienta pedagógica que debe ser adaptada al nivel educativo y madurativo del alumnado" (p. 85). Por lo tanto, es responsabilidad del profesorado adaptar cada una de las actividades de clase de acuerdo con el nivel cognitivo de su colectivo estudiantil y propiciar por medio de la gamificación un verdadero aprendizaje significativo. 
En la siguiente tabla, se recopilan las apreciaciones del estudiantado sobre las diferencias entre la gamificación y el juego. Se hace notorio que, se comprende la diferencia entre lo que sería un juego aplicado en una actividad de clase y la gamificación como un enfoque de enseñanza-aprendizaje. La introducción del juego por sí solo no significa un cambio significativo, pero si se incorporan técnicas gamificadas como los retos, el aprendizaje invertido y la autocrítica se podría llegar a un aprendizaje significativo.

\section{Tabla 2}

\section{Diferencias entre la gamificación y el juego según las apreciaciones del estudiantado del segundo nivel del BEESEC (2017)}

\begin{tabular}{|c|l|}
\hline Estudiantado & \multicolumn{1}{|c|}{ Valoraciones en torno a la gamificación } \\
\hline \multirow{4}{*}{$\begin{array}{c}\text { Segundo nivel } \\
\text { del BEESEC }\end{array}$} & $\begin{array}{l}\text { Ea gamificación es similar al juego, pero con un carácter y objetivo } \\
\text { educativo. } \\
\text { gamificación involucra el desarrollo de técnicas para producir un } \\
\text { aprendizaje. }\end{array}$ \\
\cline { 2 - 3 } & $\begin{array}{l}\text { El juego se emplea de forma aislada y el único objetivo es } \\
\text { entretener, la gamificación no se emplea de forma aislada, sino } \\
\text { que tiene todo un contexto al que responde y su objetivo va más } \\
\text { allá de entretener, tiene un objetivo formativo, que los estudiantes } \\
\text { aprendan y apliquen lo que se les está enseñando. }\end{array}$ \\
\cline { 2 - 3 } & $\begin{array}{l}\text { La gamificación tiene un fin educativo, algo que el juego muchas } \\
\text { veces no posee. }\end{array}$ \\
\hline
\end{tabular}

Fuente: Elaborado por los autores. Entrevista No. 2, ítem 4, 13 de noviembre, 2017.

Ahora bien, es importante analizar qué tipo de habilidades se fomentan a través del uso de la gamificación. De acuerdo con el estudiantado de segundo nivel, la libertad en el aprendizaje es la mayor habilidad que se potencia desde la gamificación, seguido por el trabajo colaborativo y el aprendizaje basado en retos. Se coincide con el estudiantado, al creer que la libertad del aprendizaje 
se debe propiciar desde este enfoque educativo, pero también desde cualquier escenario pedagógico donde el estudiantado debe propiciar la construcción de su aprendizaje, claro está, con el apoyo del profesorado. En el gráfico 2 se muestran las habilidades que cree el estudiantado se deben propiciar desde la gamificación en la enseñanza de la Historia:

\section{Gráfico 2}

Habilidades que se fomentan a partir de la gamificación para la enseñanza de la Historia según las apreciaciones del estudiantado del segundo nivel del BEESEC (2017)

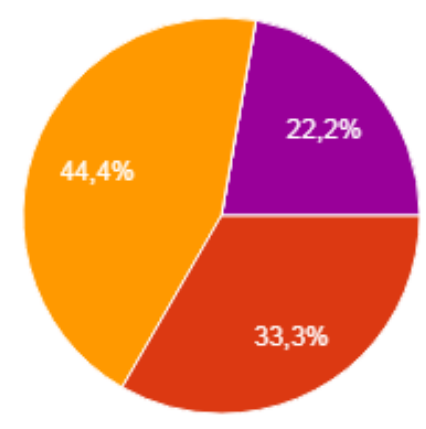

Trabajo cooperativo

Trabajo colaborativo

Libertad en el aprendizaje

Argumentación de hechos históricos

Aprendizaje basado en retos

Fuente: Elaborado por los autores. Entrevista No. 2, ítem 8, 13 de noviembre, 2017.

Por último, es importante mencionar que este enfoque se enmarca dentro de una propuesta de innovación educativa que contempla aspectos como: tecnología, didáctica y pedagogía. Para López y Heredia (2017):

Una innovación educativa implica la implementación de un cambio significativo en el proceso de enseñanza-aprendizaje, de los materiales empleados para el mismo, de los métodos de entrega de las sesiones, de los contenidos o en los contextos que implican la enseñanza (p. 18).

Según la propuesta de las autoras, la innovación implica una diferencia que se debe relacionar con la calidad y lo novedoso de aquellos elementos que se 
someten a una mejora continua en busca de llegar a entregar un aporte sustancial al proceso de enseñanza-aprendizaje. Además, proponen una escala que se caracteriza por la innovación disruptiva, que tiene la capacidad y el potencial desconocidos para afectar a todo y a todos los que actúan en el contexto educativo; la innovación revolucionaria, que muestra la aplicación de un nuevo paradigma y se revela como un cambio fundamental en el proceso de enseñanza-aprendizaje y un cambio significativo de las prácticas existentes; la innovación incremental, que se construye con base en los componentes de una estructura ya existente, dentro de una arquitectura o diseño establecido y, por último, la innovación continua, que promueve cambios que solo afectan parcial o limitadamente a los procesos o elementos del contexto educativo, o que proponen su mejora como eficiencia de operación, entrega o procedimiento (p. 18).

\section{Conclusiones}

Como parte de nuevos procesos de aprendizaje y de modificaciones en la mediación pedagógica, la gamificación se convierte en una alternativa viable y novedosa en el desarrollo de los temas de la enseñanza de la historia, ya que establece nuevos paradigmas sobre la concepción de las dinámicas y demandas que encierran las nuevas realidades en la forma de enseñar la historia. Llopis y Balaguer (2016) establecen que mediante la gamificación educativa se pretende hacer frente a la desmotivación, el aburrimiento y la falta de compromiso con la tarea; en definitiva, a aquellas situaciones y circunstancias que dificultan el aprendizaje (p. 86). Se evidencia que la experiencia de aprendizaje mediado con actividades, en la cual se utiliza el juego como recurso pedagógico y siendo este parte de la gamificación, responde a una alternativa significativa y de impacto 
dentro de la nueva cultura educativa y de mediación que se potencia en la sociedad actual.

El profesorado de Historia debe prepararse para cumplir con los retos que exige una mediación más dinámica y entretenida. Debe abandonar el viejo paradigma y pensar en una innovación educativa que se ajuste a las competencias que se promueven en la actualidad. Es decir, el docente en historia debe poseer un alto dominio de una serie de competencias con el fin de generar espacios de aprendizaje, donde el estudiante pueda adoptar y crear diferentes perspectivas del objeto de estudio y generar alternativas explicativas de la realidad, con el desarrollo de pensamiento crítico y comprometido, y en particular, potenciar el uso pedagógico de las tecnologías de la información.

Se puede señalar que la gamificación es una alternativa real y significativa para generar aprendizajes, ya que puede potenciar el desarrollo del trabajo cooperativo, el trabajo colaborativo, la libertad en el aprendizaje, la argumentación de hechos históricos, el aprendizaje basado en retos, entre otros. Lo que facilita a la vez en los docentes una serie de retos, debido a la necesidad de un cambio de roles y la redefinición de paradigmas sobre la mediación pedagógica y la claridad en relación con la necesidad de introducirse en la alfabetización de la mediación con ayuda de los medios tecnológicos de la información, es decir, se requiere de un nuevo docente que sea capaz de visualizar su rol de mediador y facilitador de procesos reales de aprendizaje, acordes a la realidad actual y cambiante. 


\section{Bibliografía}

Collazo, H. \& de Ranalletti, V. (2015). "Las TIC en la formación presencial y a distancia de futuros y jóvenes docentes para la enseñanza de la historia". Memorias (0124-4361), 13(24), 1-22, DOI: https://doi.org/10.16925/me.v13i24.1538

EduTrends. (2016). "Gamificación”. Tecnológico de Monterrey, Observatorio de Innovación Educativa.

Entrevista con el estudiantado de cuarto nivel del BEESEC. (4 de octubre de 2017). Indagaciones sobre proyectos de innovación educativa [Entrevista No. 1]. Disponible en URL: https://goo.gl/forms/8VyNAvnZ6sDMGify1

Entrevista con el estudiantado de segundo nivel del BEESEC. (13 de noviembre de 2017). Valoraciones sobre la gamificación en la Enseñanza de los Estudios Sociales y Educación Cívica. [Entrevista No. 2]. Disponible en URL: https://goo.gl/forms/GNHOykPZCupJ2f2P2

Fallas, V. \& Trejos, I. (2013). Educación en la sociedad de la información y el conocimiento. San José, Costa Rica: EUNED.

Fandos, M. \& González, A. (2013). "La «gamificación» como motivación en los entornos virtuales de aprendizaje". Educamed, noviembre 2013 (pp. 18). Barcelona, España: UOC, DOI: https://doi.org/10.13140/2.1.1902.6568

Feliu, M., Vallés, A. \& Cardona, G. (2016). "Innovación en la formación inicial del profesorado: exploración de creencias sobre la enseñanza de las Ciencias Sociales en alumnos de Magisterio". Revista Electrónica Interuniversitaria De Formación Del Profesorado, 19(3), 1-9, DOI: https://doi.org/10.6018/reifop.19.3.267221

Ferreiro, R. \& Vizoso, E. (2008). "Una condición necesaria en el empleo de las TICs en el salón de clases: la mediación pedagógica". Revista Posgrado y Sociedad, 8(2), 72-88. 
González, M., Pagès, J. \& Santisteban, A. (2009). Proyecto de Investigación financiado por el Ministerio de Ciencia e Innovación de España. Referencia: EDU2009-10984. Período: 2009-2012. Investigador Principal: Antoni Santisteban.

Llopis, M.a A. \& Balaguer, P. (2016). "El uso del juego en educación. Gamificación". En: Métodos pedagógicos activos y globalizadores. Conceptualización y propuesta de aplicación (pp. 85-102). Barcelona: GRAÓ.

Taracena, L. (2015). "De la historia aburrida a la entretenida". Perspectivas. Revista de Estudios Sociales y Educación Cívica, (10), 10-41, URL: http://www.revistas.una.ac.cr/index.php/perspectivas/article/view/6749 